\title{
RADIOCARBON INTERCOMPARISON STUDIES AT THE RUDJER BOŠKOVIĆ INSTITUTE
}

\author{
INES KRAJCAR BRONIĆ, NADA HORVATINČIĆ, BOGOMIL OBELIĆ and \\ ROMANA BRISTROVIĆ
}

Rudjer Bošković Institute, P.O. Box 10001 Zagreb, Croatia

\begin{abstract}
The Radiocarbon Tritium Laboratory at the Rudjer Boßkovic Institute participated in all three radiocarbon intercomparison studies. The first International Collaborative Study (ICS) showed that the age of organic samples measured at our laboratory is $c a$. 70 yr younger than the median ICS value. After introducing a slight modification in our chemical pretreatment procedure by acidifying samples after alkaline treatment, we obtained excellent agreement between our results and the median values reported by two subsequent intercomparisons (IAEA, TIRI) without any laboratory bias.
\end{abstract}

\section{INTRODUCTION}

The Rudjer Boškovic Institute (RBI) Radiocarbon Laboratory participated in three recent ${ }^{14} \mathrm{C}$ intercomparison studies. The first International Collaborative Study (ICS) was organized by the Department of Statistics, University of Glasgow. Horvatincic et al. (1990) reported and discussed the results of our measurements at the International Workshop on Intercomparison of Radiocarbon Laboratories, together with all the other major findings of the ICS (Long, Scott and Kra 1990). The second intercomparison was organized by the International Atomic Energy Agency (IAEA), and Rozanski et al. (1992) reported the consensus values for five unknown samples and ANU Sucrose. The Third International Radiocarbon Intercomparison (TIRI), organized again by the Department of Statistics, University of Glasgow in 1992, was divided into two stages (Scott et al. 1992). Stage 1 included six core samples identical for all participating laboratories, whereas in Stage 2, each laboratory requested optional samples provided by the organizer.

\section{METHODS}

The sample preparation and measurement technique used in our laboratory is essentially the same as described in Srdoč, Breyer and Sliepčević (1971), supplemented by computerized data processing (Obelić 1989). Calcareous samples are dissolved in $\mathrm{HCl}$. Chemical pretreatment of organic samples includes boiling water in $4 \% \mathrm{HCl}$, neutralization, and boiling in a $4 \%$ solution of $\mathrm{NaOH}$ and additional boiling of $4 \% \mathrm{HCl}$. Organic samples are combusted in a stream of purified oxygen. $\mathrm{CO}_{2}$ is hydrogenated to $\mathrm{CH}_{4}$ over a Ru catalyst. A gas-proportional counter filled with purified methane is used for ${ }^{14} \mathrm{C}$ activity measurement. Each sample is routinely measured twice, or even several times, and the mean value is calculated and reported. The age is calculated following the recommendations by Stuiver and Polach (1977). We also apply the fractionation correction for $\delta^{13} \mathrm{C} .{ }^{1}$

We measured most of the intercomparison samples immediately after methane preparation, to detect any radon that might be present, by monitoring the sample activity over a period of $30-40$ days. We found a relatively high radon contamination in TIRI sample $H$ (Ellanmore peat) and TIRI sample $L$ (whale bone) (Fig. 1). Fitting the mean daily count rate for both samples to an exponential curve resulted in half-lives of 4 and 3.5 days, respectively, close to the half-life of ${ }^{222} \mathrm{Rn}$ ( 3.82 days). We also detected small amounts of radon in several other samples. Horvatinčic et al. (1995) present a more comprehensive study of radon contamination in various kinds of samples used in ${ }^{14} \mathrm{C}$ dating.

${ }^{1} \delta^{13} \mathrm{C}$ values of the samples were measured at the Jožef Stefan Institute, Ljubljana, Slovenia. 


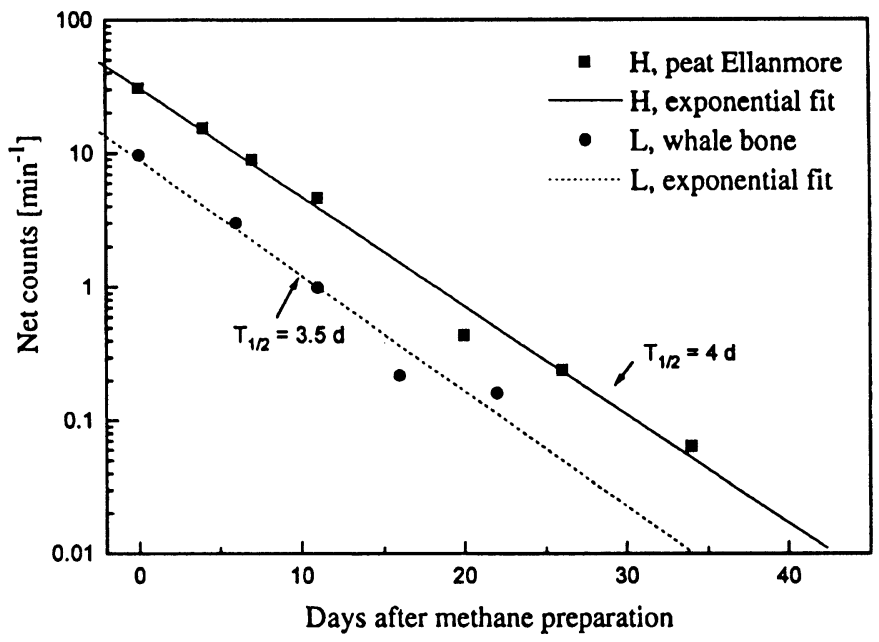

Fig. 1. Mean daily net count rates of TIRI Stage 1 samples $\mathrm{H}$ and $\mathrm{L}$, as a function of the number of days elapsed since methane preparation. Count rates were fitted by exponential functions and the half-lives were determined.

\section{RESULTS}

We present the summary of our results and the median (consensus) values of all three intercomparison studies in Table 1 . We were not able to compare all the data in a single plot, because the median values of the three intercomparison studies were not presented in the same format: the median values published in the first ICS study were given in years BP, the consensus values of the IAEA intercomparison in pMC, and the TIRI values in $\mathrm{D}^{14} \mathrm{C}$ and either pMC (modern samples, $\mathrm{pMC}>100 \%$ ) or in years BP. In Figures 2, 4 and 5, we compare our measured data expressed in years BP, pMC values and $\mathrm{D}^{14} \mathrm{C}$ with the median (consensus) values for ICS, IAEA and TIRI samples, respectively.

The linear regression line $y=k x+l$ was calculated for all three figures, where $x$ represents the RBI measured data, and y represents the median/consensus value. The values of coefficient $k$ and $l$ should be 1 and 0 for ideal agreement and no laboratory bias.

In the first ICS study, we measured 16 samples, obtaining the following linear regression line (Fig. 2):

$$
\operatorname{age}_{\mathrm{ICS}}=(1.00 \pm 0.02) \mathrm{age}_{\mathrm{RBI}}+(70 \pm 42), \mathrm{n}=16, \mathrm{r}=0.997 \text {. }
$$

As we discussed previously (Horvatincić et al. 1990), our measurements averaged ca. 70 yr younger than the median ICS values. Although inorganic (carbonate) samples deviated randomly (except sample S, as discussed in Horvatinčić et al. (1990)), organic samples clustered at ca. 1-2 o (standard deviations) below the ICS median values. In Figure 3, we present the relative deviations of our results from the median values in the form of bar graphs, where the clustering of the ICS organic samples (top) is clearly seen. This systematic bias was discussed at the Glasgow International Workshop (Scott, Long and $\mathrm{Kra} 1990$ ), and it was pointed out that our two-step chemical pretreatment by boiling samples in diluted $\mathrm{HCl}$ and $\mathrm{NaOH}$ could have introduced contamination resulting in younger sample ages. Since then, we have slightly modified our chemical pretreatment procedure by adding a third step consisting of acidification by boiling samples for a short time in very dilute $\mathrm{HCl}$, washing to neutral and drying. The additional acidification of organic samples helped to avoid possible contamination via absorption of atmospheric $\mathrm{CO}_{2}$ by traces of $\mathrm{NaOH}$ in which the samples were boiled. 
TABLE 1. Comparison of the Rudjer Bošković Institute Radiocarbon Laboratory Measurements of All Intercomparison Samples with the Median Values of the ICS, IAEA and TIRI Intercomparison Studies

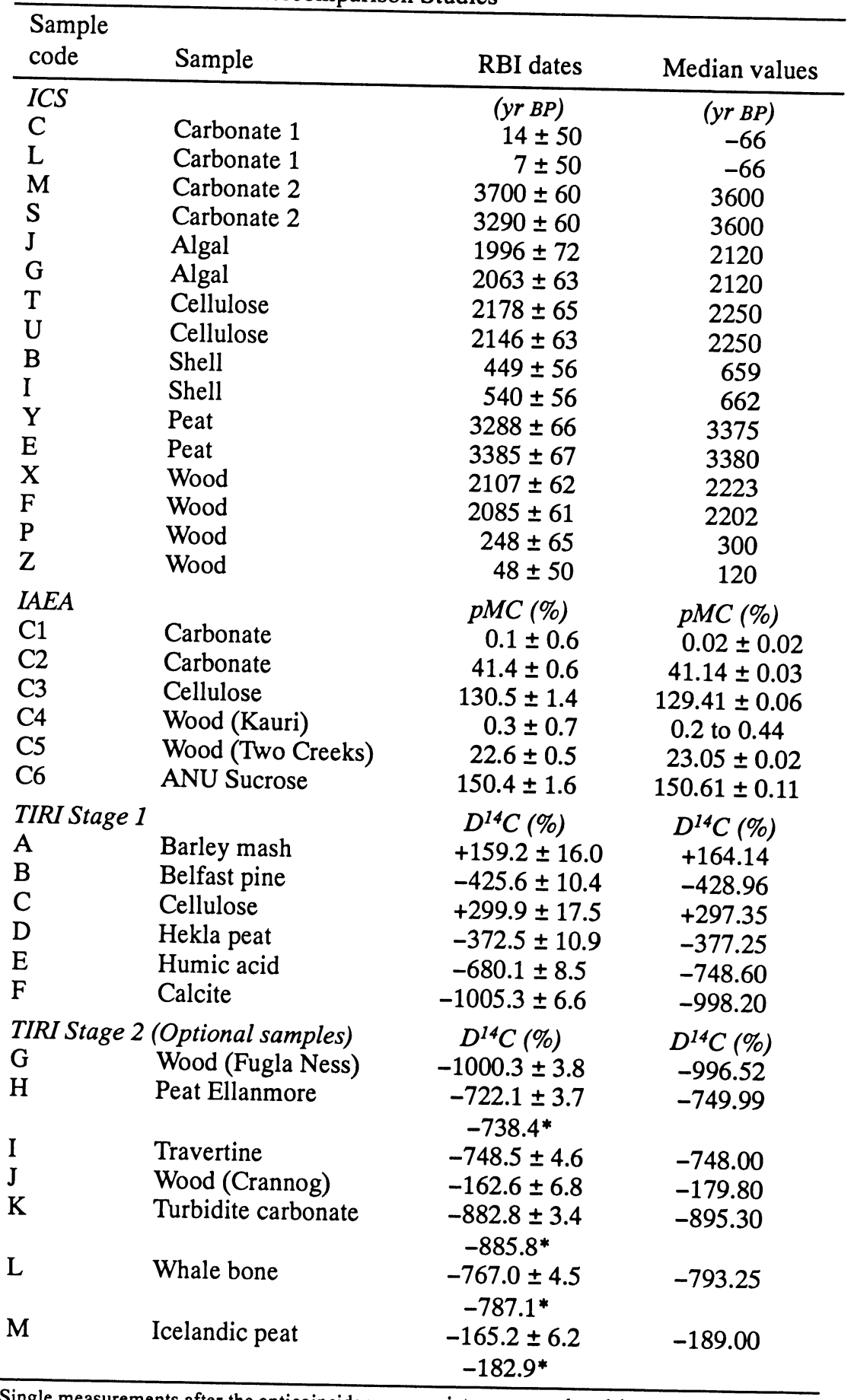

*Single measurements after the anticoincidence gas mixture was replaced (see text) 


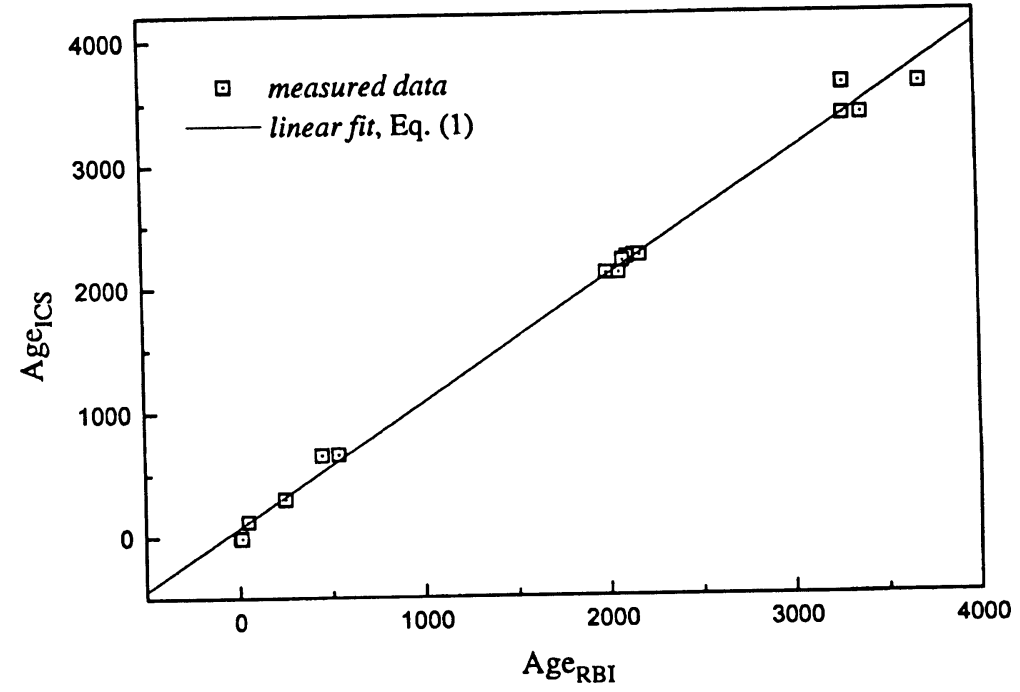

Fig. 2. Comparison of ICS sample ages as measured at the Rudjer Boß̌kovic Institute Radiocarbon Laboratory (on abscissa) and the ICS median values. Equation (1) in text gives the linear regression line.
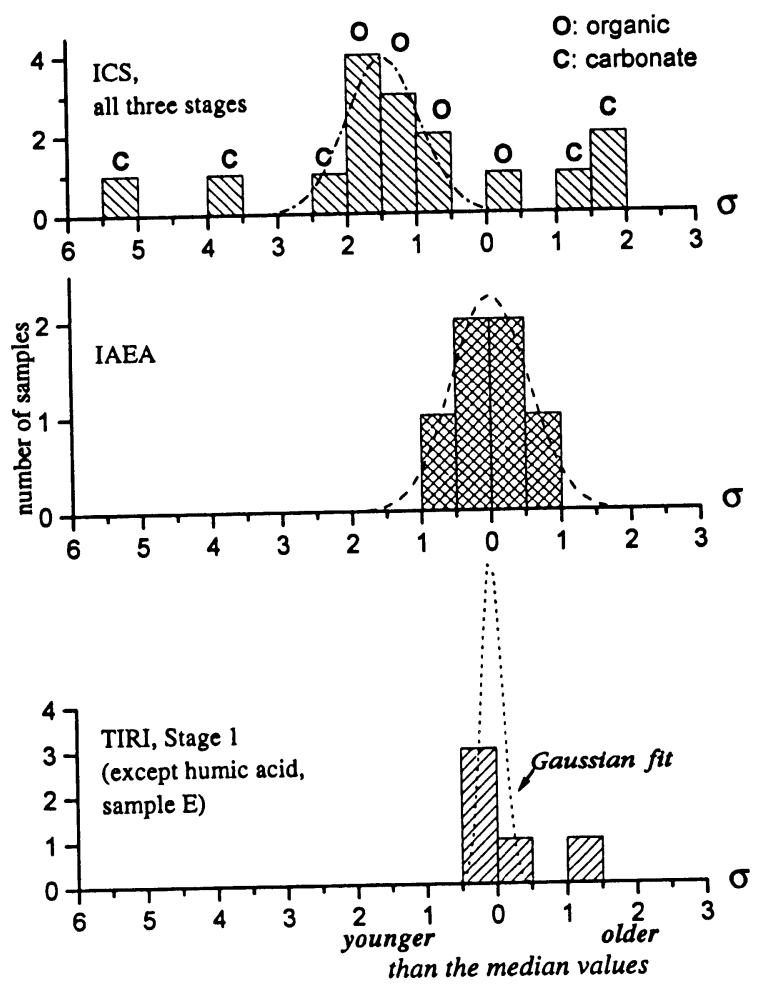

Fig. 3. Relative deviations, calculated as the difference between the RBI results and the median values divided by the standard deviation of the $\mathrm{RBI}$ result: (result $\mathrm{RBI}-$ median) / $\sigma_{\mathrm{RBI}}$, for the three intercomparison studies 
Figure 4 shows our IAEA intercomparison data. The regression line follows the equation

$$
\mathrm{pMC}_{\mathrm{IAEA}}=(1.00 \pm 0.04) \mathrm{pMC}_{\mathrm{RBI}}+(0.0+0.3), \mathrm{n}=6, \mathrm{r}=0.999 \text {. }
$$

All our results with $1 \sigma$ interval included the consensus values, and we observed no systematic bias (Fig. 3, center).

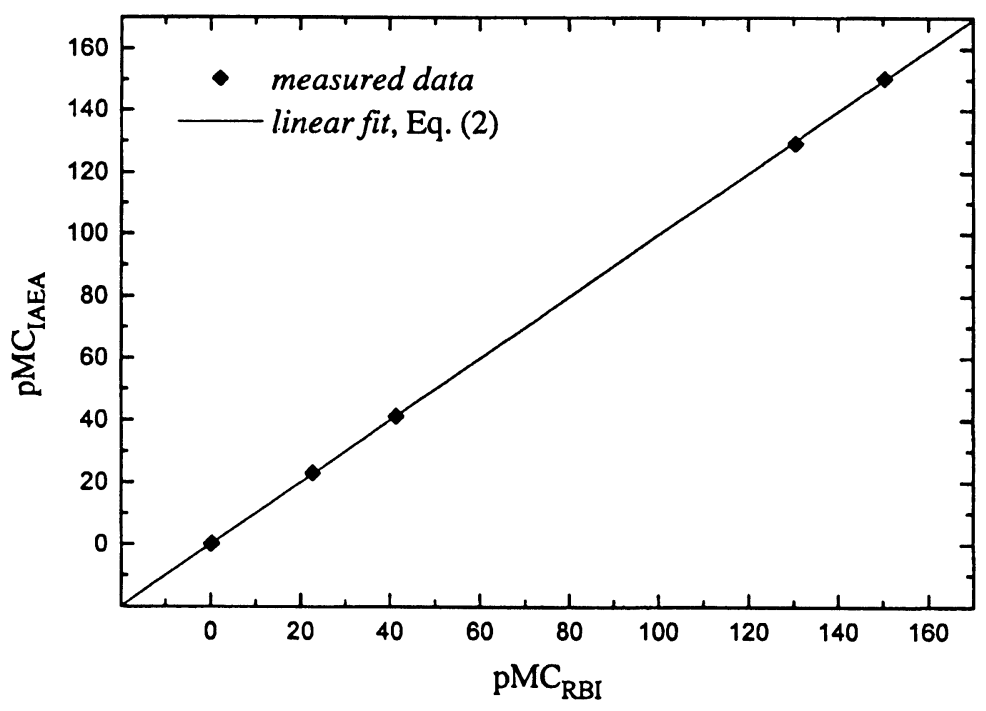

Fig. 4. Comparison of the IAEA sample activities as measured at the Rudjer Boskovic Institute Radiocarbon Laboratory (on abscissa) and the IAEA median values. Equation (2) in text gives the linear regression line.

For TIRI Stage 1 core samples (Fig. 5), we obtained the following regression line between the median TIRI $\mathrm{D}^{14} \mathrm{C}$ values and our $\mathrm{D}^{14} \mathrm{C}$ values:

$$
\mathrm{D}^{14} \mathrm{C}_{\mathrm{TTRI}}=(1.01 \pm 0.02) \mathrm{D}^{14} \mathrm{C}_{\mathrm{RBI}}+(-5.7 \pm 15.6), \mathrm{n}=6, \mathrm{r}=0.998 \text {. }
$$

The differences between the measured data and the TIRI median values for 5 out of 6 samples were $<1 \sigma$ (Fig. 3, bottom), and only for sample $\mathrm{E}$ (humic acid) was the difference significant. Because this sample was limited in quantity, we did not pretreat it before combustion; this was also the first time that we measured humic acid in our laboratory. If we exclude this outlier, we obtain the following regression (Fig. 5, solid line):

$$
\mathrm{D}^{14} \mathrm{C}_{\mathrm{TRI}}{ }^{*}=(0.997 \pm 0.005) \mathrm{D}^{14} \mathrm{C}_{\mathrm{RBI}}{ }^{*}+(-6.0 \pm 3.0), \mathrm{n}=5, \mathrm{r}=0.999 \text {. }
$$

The graphs in Figure 3 show that the IAEA and TIRI Stage 1 intercomparison data lie within $\pm 1 \sigma$, indicating very good agreement between the measured results with the standard deviations reported and the median values, with no systematic bias.

For TIRI Stage 2 samples (Fig. 5, dashed line) we obtained the following regression line between the median TIRI $\mathrm{D}^{14} \mathrm{C}$ values and our $\mathrm{D}^{14} \mathrm{C}$ values:

$$
\mathrm{D}^{14} \mathrm{C}_{\mathrm{TRR} \mathrm{I}_{2}}=(0.983 \pm 0.015) \mathrm{D}^{14} \mathrm{C}_{\mathrm{RBI}}+(-26 \pm 10), \mathrm{n}=7, \mathrm{r}=0.999 \text {. }
$$

The correlation shows again that our results are, on average, much higher than the median TIRI values, although we performed the complete treatment method. We found that the systematic bias for 


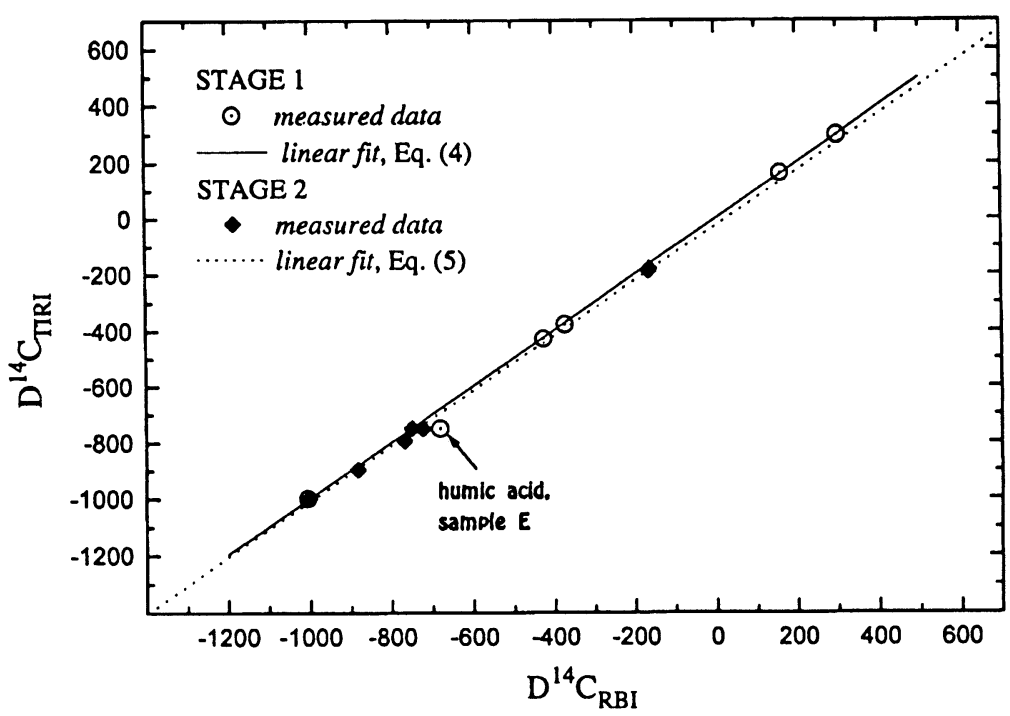

Fig. 5. Comparison of the TIRI sample activities (Stages 1 and 2) as measured at the Rudjer Boskovic Institute Radiocarbon Laboratory (on abscissa) and the TIRI median values. The data point for sample $\mathrm{E}$ (Stage 1) was not taken into account in calculating the linear regression line, Equation (4) in text. Equation (5) in text gives the linear regression line $(\cdot . \cdot)$ ) for TIRI Stage 2 samples.

this series was caused by poor gas mixture used in the anticoincidence GM counter. We replaced the gas mixture and repeated measurement of five TIRI Stage 2 samples (Table 1, results denoted by asterisks). The deviations from the TIRI median values are now much smaller and the following correlation is obtained:

$$
\mathrm{D}^{14} \mathrm{C}_{\mathrm{TRR}_{2}}{ }^{*}=(1.002 \pm 0.014) \mathrm{D}^{14} \mathrm{C}_{\mathrm{RBI}}{ }^{*}+(-10.7 \pm 8.8), \mathrm{n}=5, \mathrm{r}=0.999 \text {. }
$$

\section{CONCLUSION}

The first intercomparison study showed that the ages of organic samples, as measured at the RBI Laboratory, were slightly younger than the reported ICS median values. The additional acidification of organic samples in the chemical pretreatment procedure prevented possible contamination by absorption of atmospheric $\mathrm{CO}_{2}$ after alkaline treatment of samples. The modified three-step acidbase-acid chemical pretreatment procedure thus suppressed a systematic bias in the subsequent IAEA and TIRI intercomparisons. Relatively large deviations of our results from the TIRI Stage 2 median values were significantly reduced after replacing the old gas mixture in the anticoincidence GM counter.

\section{ACKNOWLEDGMent}

This work was supported by Grant No. 1-07-064 from the Ministry of Science, Republic of Croatia. 


\section{REFERENCES}

Horvatinđiç, N., Obelić, B., Krajcar Bronic, I., Srdoð, D. and Bistrovic, R. 1995 Sources of radon contamination in ${ }^{14} \mathrm{C}$ dating. Radiocarbon, this issue.

Horvatincic, N., Srdoc, D., Obelic, B. and Krajcar Bronic, I. 1990 Radiocarbon dating of intercomparison samples at the Zagreb Radiocarbon Laboratory. In Scott, E. M., Long, A. and Kra, R. S., eds., Proceedings of the International Workshop on Intercomparison of Radiocarbon Laboratories. Radiocarbon 32(3): 295-300.

Obelic, B. 1989 The radiocarbon data base at Rudjer Boß̌kovic Institute Radiocarbon Laboratory. In Long, A., Kra, R. S. and Srdoc, D., eds., Proceedings of the 13th International ${ }^{14} \mathrm{C}$ Conference. Radiocarbon 31(3): 1057-1062.

Rozanski, K., Stichler, W., Gonfiantini, R., Scott, E. M., Beukens, R. P., Kromer, B. and van der Plicht, J. 1992 The IAEA Intercomparison Exercise 1990. In Long,
A. and Kra, R. S., eds., Proceedings of the 14th International ${ }^{14} \mathrm{C}$ Conference. Radiocarbon 34(3): 506519.

Scott, E. M., Harkness, D. D., Miller, B. F., Cook, G. T. and Baxter, M. S. 1992 Announcement of a further International Intercomparison Exercise. In Long, A. and Kra, R. S., eds., Proceedings of the 14th International ${ }^{14} \mathrm{C}$ Conference. Radiocarbon 34(3): 528-532.

Scott, E. M., Long, A. and Kra, R. S., eds., 1990 Proceedings of the International Workshop on Intercomparison of Radiocarbon Laboratories. Radiocarbon 32(3): 253-397.

Srdoc, D., Breyer, B. and Sliep̌evic, A. 1971 Rudjer Boskovic Institute radiocarbon measurements I. Radiocarbon 13(1): 135-140.

Stuiver, M. and Polach, H. A. 1977 Discussion: Reporting of ${ }^{14} \mathrm{C}$ data. Radiocarbon 19(3): 355-363. 